\title{
Intracranial space-occupying masses in mental hospital patients: necropsy study
}

\author{
G I L L I A N C O L E
}

From the Department of Neuropathology, School of Pathology, South African Institute for Medical Research and University of Witwatersrand, Johannesburg, South Africa

SUM MARY During a three year period 1970-1973 necropsies were performed on 200 patients who died in a mental hospital in the Transvaal, South Africa. There was no selection of cases other than availability of consent for postmortem examination. The necropsy rate for this period was $56 \%$. The patients belonged to three racial groups, Black, White, and Coloured, the latter being defined as those of mixed blood. Among these 200 cases, 27 were found at necropsy to have an intracranial space-occupying mass. Mention is made of the literature dealing with the incidence and diagnosis of intracranial space-occupying masses in mental hospital patients, and the incidence of intracranial space-occupying masses in the different racial groups in South Africa.

The few figures available on the incidence of intracranial space-occupying masses in mental hospital patients reflect a higher incidence of cerebral neoplasms in psychiatric patients (Andersson, 1970). Most investigators have included neoplasms, granulomas, abscesses, and subdural haematomas under the general heading of intracranial spaceoccupying masses. There is a scarcity of literature regarding both the incidence and antemortem recognition of subdural haematomas in mental hospital patients.

In South Africa the incidence of intracranial neoplasms in Blacks has been found to be lower than it is in Whites. Proctor (1955) found that astrocytomas and meningiomas together were five times more common in the White than in the Black. Other space-occupying lesions were scrutinised, and tuberculomas were found to be 10 times and parasitic cysts twice as common in the Black patient. Brain abscess occurred with about equal frequency in the two racial groups, and metastatic brain lesions were three times more common in the White patient. A survey of 3545 Black patients with neurological disease admitted to Baragwanath Hospital over one year revealed that among these cases there were 17 neoplasms and 42 subdural and extradural haemorrhages (Reef et al., 1958). A low incidence of cerebral

Address for reprint requests: Dr Gillian Cole, c/o SAIMR Library, PO Box 1038, Johannesburg 2000, South Africa.

Accepted 23 February 1978 neoplasms was found by Froman and Lipschitz (1970) who studied the incidence of primary central nervous system tumours over a 20 year period in the Black population of the Transvaal, and found it to be $0.026 \%$ of hospital admissions in the last five year period.

Available figures for the proportion of undiagnosed intracranial space-occupying masses vary from 31 to $83 \%$ with an average of $55 \%$ (Selecki, 1964). From analysis of 5862 mental hospital cases Andersson (1970) gave a figure of $65 \%$.

The diagnosis of an organic brain lesion may be difficult to make when mental symptoms are prominent. Numerous cases are cited where the clinical presentation is of a psychiatric nature with little or nothing in the way of neurological signs until a late stage. Whether an intracranial mass is developed in the presence of a psychosis, or a psychosis develops as a result of it, the mental symptoms may effectively mask the presence of an organic lesion. If a psychotic patient develops an intracranial mass additional symptoms may go unnoticed or not be complained of. No truly specific mental symptoms are likely to help in the diagnosis although Selecki (1964) has drawn attention to the frequency with which they are found in midline tumours.

\section{Material and methods}

In the present study, I carried out a full routine 
postmortem examination on each case. The brain was removed after inspection of the scalp, skull, and dura mater, and suspended by the basilar artery in $10 \%$ formalin for a minimum of three weeks. When the brain was fixed, it was inspected, and after removal of the hind brain, the cerebrum was sliced in coronal sections at $10 \mathrm{~mm}$ intervals. Routine sections for histological examination were taken from the superior frontal gyrus, precentral gyrus, cuneate gyrus, parietal lobe, left and right hippocampus with temporal lobe cortex and inferior horn of lateral ventricle, basal ganglia, cerebellar cortex and dentate nucleus, midbrain, pons, and medulla. Additional sections were taken from the brain where any pathology was macroscopically evident. Sections were cut at $4 \mu \mathrm{m}$ embedded in paraffin and routinely stained with haematoxylin and eosin, Kluver Barrera stain for myelin and Nissl substance, and phosphotungstic acid haematoxylin for the demonstration of gliosis. Other stains such as von Braunmühls silver stain were used as required. Sections were taken routinely from liver, heart, kidney, and lung, and any other organ where pathological change was evident. Routine haematoxylin and eosin stains on sections of $4 \mu \mathrm{m}$ were examined, and where indicated other special stains such as Masson's and iron stains in cases with cirrhosis of the liver. The findings on histological examination together with a summary of the clinical history and diagnosis were recorded on a pro forma.

\section{Results}

Twenty-seven cases with an intracranial spaceoccupying mass were found $(13.5 \%$ of the necropsy material). The type of mass and racial distribution are summarised in Table 1.

\section{SUBDURAL HAEMATOMA}

In this investigation the most common type of intracranial mass was a subdural haematoma. There were 14 cases which constituted $7 \%$ of the

Table 1 Type of mass found and racial distribution of patients

\begin{tabular}{lcccc}
\hline & \multicolumn{2}{l}{ Racial group } & \\
\cline { 2 - 5 } Intracranial mass & White & Black & Coloured & Total \\
\hline Subdural haematoma & 3 & 10 & 1 & 14 \\
Primary neoplasm & 3 & 1 & - & 4 \\
Metastatic neoplasm & - & 1 & - & 1 \\
Hamartoma & - & 1 & - & 1 \\
Tuberculoma & - & 3 & - & 3 \\
Parasitic tapeworm cyst & 1 & 1 & - & 2 \\
Abscess & 1 & 1 & - & 2 \\
\hline
\end{tabular}

necropsy material. The incidence in the three racial groups is indicated in Table 2 .

Type of haematoma There were six cases each of chronic and acute subdural haematoma, and two cases which were classified as subacute (1-2 weeks of age).

Table 2 Incidence of subdural haematoma by racial group

\begin{tabular}{lcc}
\hline Racial group & \multicolumn{2}{c}{ Number of subdural haematomas } \\
\hline Black & $10 / 121$ & $8.1 \%$ \\
Coloured & $1 / 15$ & $6.6 \%$ \\
White & $3 / 64$ & $4.6 \%$ \\
\hline
\end{tabular}

Acute subdural haematoma of the six cases in this group five were Black (four males and one female) and one was a Coloured male patient. The age of death ranged from 35-55 years. The duration of hospitalisation before death was short, ranging from one to four days in five of the patients. At necropsy evidence of recent head injury was found in five cases, and this was in the form of small lacerations and bruising of the scalp, which in pigmented skin was more evident on the inner surface of the scalp. An old fracture was present in one case with the antemortem diagnosis of post-traumatic dementia, and this was the only case in the acute group with cortical atrophy. In all six cases the cause of death was attributed to the haematoma.

Chronic and subacute subdural haematoma There were eight cases in this group of whom five were Black and three were White. The results of a chronic subdural haematoma in one patient is shown in Fig. 1. The patients in this group were older than those with an acute subdural haematoma, and the age at death ranged from 59-80 years. Duration of hospitalisation before death was longer than with acute haematomas, and ranged from several months to years. There was only one case with any evidence of a head injury and in this case there was a history of a recent fall. Old post-traumatic cerebral scarring was present in one case, and the age of the haematoma was considered to be more than six months which suggested that this might have occurred at the same time as the injury. In two cases localised chronic meningitis was present in relation to the site of the haematoma. Cortical atrophy was present in six of the eight cases. Death was attributed to the presence of the haematoma in six cases, and was considered to have contributed to the cause of death in the remaining two patients. Development of subdural haematomas From knowledge of the duration of hospitalisation and 


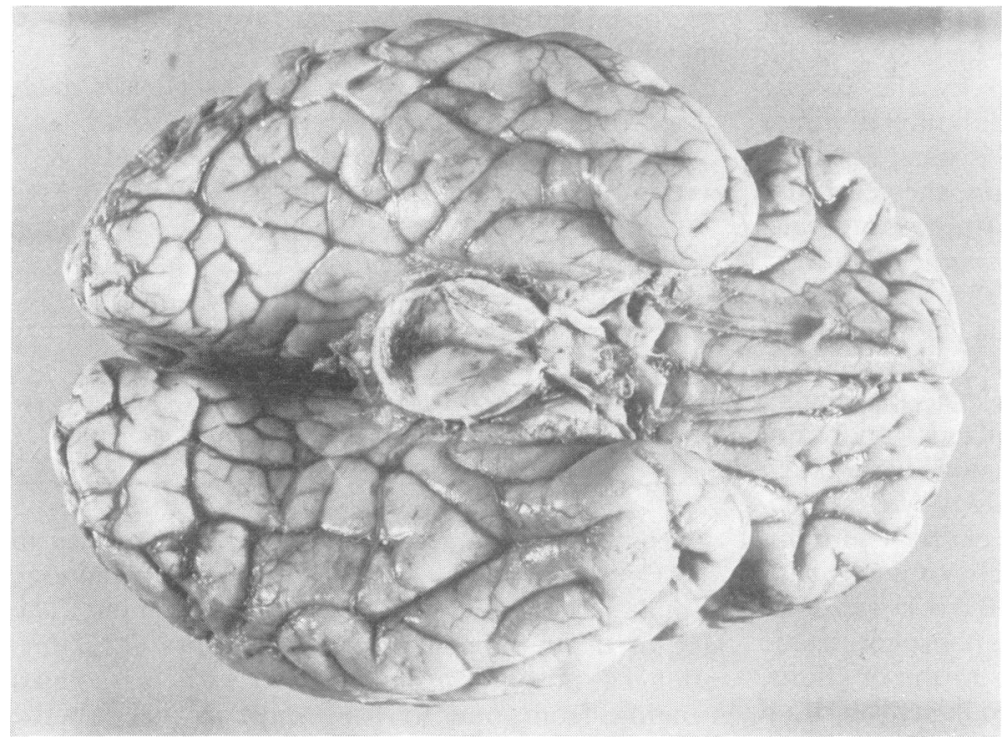

Fig. 1 Chronic subdural haematoma in a White female aged 54 yr. Note bilateral uncal herniation and secondary brainstem haemorrhages.

assessment of the approximate age of the haematoma it was possible in most cases to establish whether the haematoma developed in hospital or before admission. Among the chronic and subacute haematomas six out of eight cases developed their lesion during hospitalisation. Among the six cases with an acute subdural haematoma there were five who had a very short stay in hospital before death, and were either admitted with the lesion or it was incurred shortly after admission. In only one acute case did the lesion clearly develop long after the patient's admission to hospital with post-traumatic dementia.

Antemortem diagnosis In only one of 14 cases was the diagnosis of subdural haematoma made before death, and that case was admitted in coma with severe bruising of the scalp and face.

\section{CEREBRAL NEOPLASMS}

At necropsy there were six patients with cerebral neoplasm ( $3 \%$ of the necropsy material). The type of neoplasm and race of the patient are shown in Table 3, and the result in one patient in Fig 2 . The cause of death was directly related to the

Table 3 Type of neoplasm by racial group

\begin{tabular}{lll}
\hline & \multicolumn{2}{l}{ Racial group } \\
\cline { 2 - 3 } Type of Neoplasm & White & African \\
\hline $\begin{array}{l}\text { Oligodendroglioma } \\
\text { Meningioma }\end{array}$ & 2 & - \\
Glioblastoma multiforme & 1 & - \\
Metastatic carcinoma & - & 1 \\
Angiolipoma (hamartoma) & - & 1 \\
\hline
\end{tabular}

presence of cerebral neoplasm in five cases.

Development of neoplasm From consideration of the type of neoplasm and the clinical history it was concluded that all cases were admitted to hospital with the neoplasm already present.

Antemortem diagnosis The only case diagnosed before death was a patient referred from the neurosurgical unit with mental symptoms. Biopsy of the neoplasm had revealed a glioblastoma multiforme.

\section{TUBERCULOMA}

There were three Black patients with a tuberculoma and in all three cases this was associated with tuberculous meningitis. Miliary tuberculosis was present in two cases but in the third, only the brain was available for examination. In all cases the cause of death was considered to be directly related to the tuberculous infection. None of the cases were referred to hospital more than 10 days before death. In two cases on admission the correct diagnosis was considered a possibility and specific treatment had been instituted.

\section{CEREBRAL TAPEWORM CYSTS}

There were two patients (one Black and one White) in whom the postmortem diagnosis of cerebral tapeworm cyst was made. A tapeworm cyst in the brain of one of the patients is illustrated in Fig. 3. Both patients were epileptics. In neither case was the presence of tapeworm cysts considered to be the direct cause of death. The cause of death was attributed to status epilepticus in one case, and cardiovascular disease in the other. 


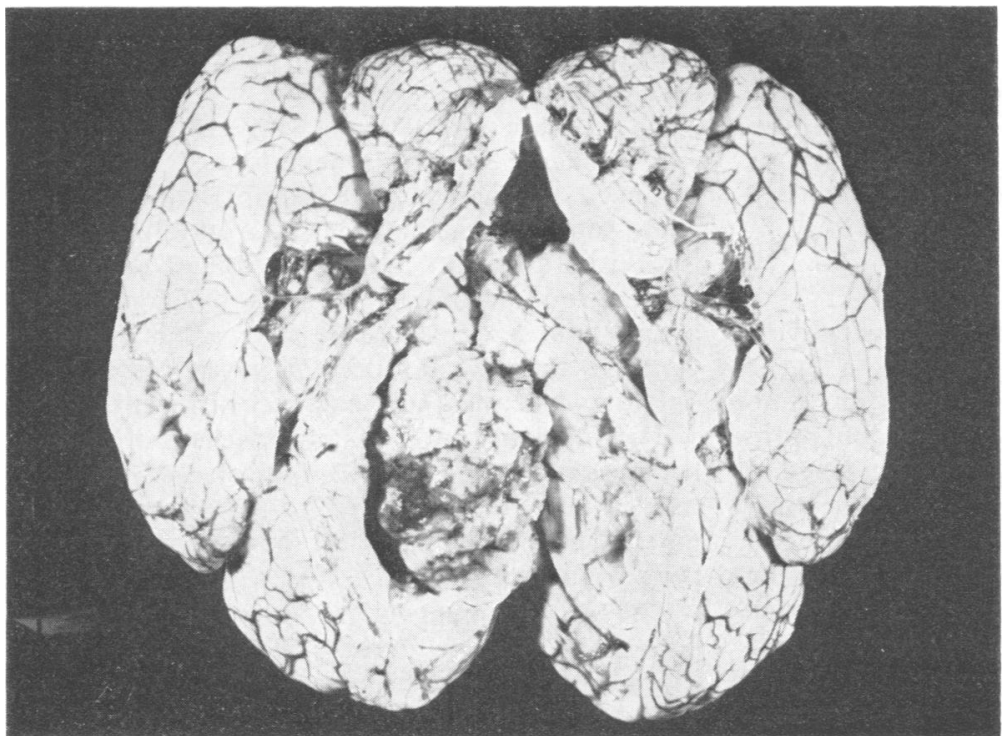

Fig. 2 Large midline tumour (oligodendroglioma) in a White male aged $34 \mathrm{yr}$.

INTRACRANIAL ABSCESS

Intracranial abscess was found in two cases at necropsy. In both cases the cause of death was attributed to the abscess. The correct diagnosis was made antemortem in one patient who developed the lesion after admission to hospital. The other case was admitted with a chronic cerebral abscess which caused death on the fourth day in hospital.

\section{PSYCHIATRIC DIAGNOSIS}

Among the group of 27 patients with an intracranial space-occupying mass there were eight patients who could definitely be stated to have developed their lesion in hospital, and therefore in the presence of a psychosis. The psychiatric diagnosis in these eight cases included three of senile dementia and one case each of manic depressive psychosis, chronic alcoholism, posttraumatic dementia, neurosyphilis, and defective mental development. The intracranial lesion was a subdural haematoma in all save one patient who developed a brain abscess. Additional mental symptoms were noted to have developed in three cases, and neurological signs of some kind were observed in five.

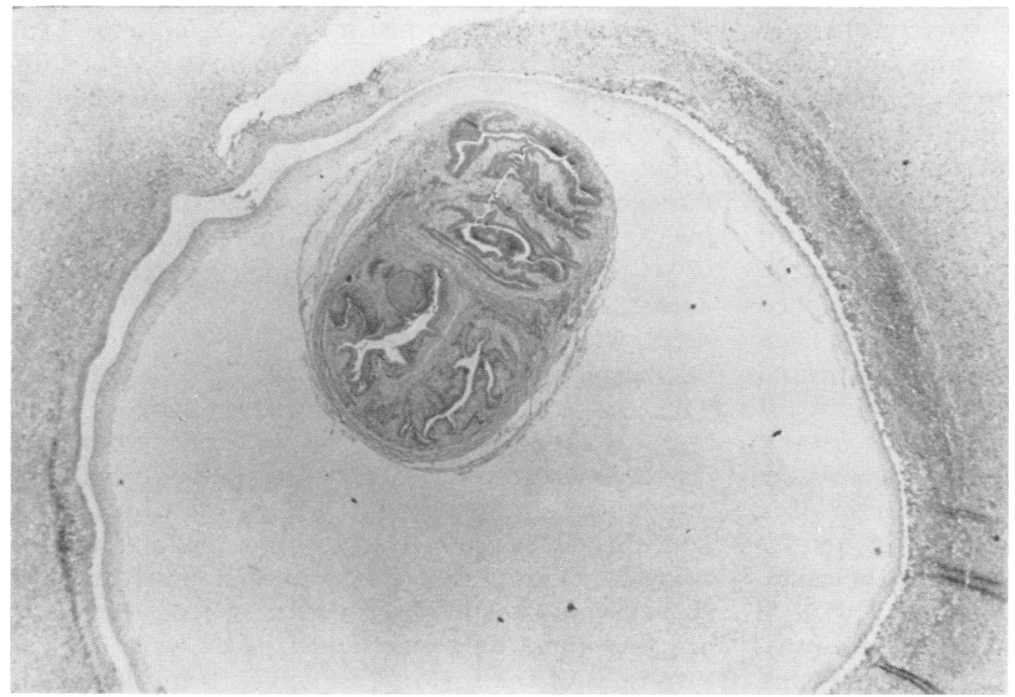

Fig. 3 Cerebral tapeworm cyst in a Black female. A cyst containing a viable worm is present in the temporal cortex. ( $H$ and $E \times 10$ ) 
There were 19 cases admitted with an intracranial space-occupying mass already present. All had mental symptoms and the psychiatric diagnosis on admission included toxic psychosis, schizophrenia, epilepsy, senile and arteriosclerotic brain disease, and organic brain disease.

\section{Discussion}

The incidence of intracranial space-occupying masses in this investigation was $13.5 \%$, a figure which is higher than most quoted for similar material in mental hospital populations (Andersson, 1970). The relatively high incidence of subdural haematomas found in the present study made a significant contribution to the number of space-occupying masses. This is contrary to the findings of most investigators who cite cerebral neoplasm as the most frequent lesion. Selecki (1965) has commented on the scarcity of literature on the incidence of subdural haematomas in mental patients. Selecki found an incidence of $1.6 \%$, but in an earlier and larger investigation Allen et al. (1940) studied 3100 psychiatric necropsy pro formas and found 245 subdural haematomas $(7.9 \%)$ which comes closer to the incidence found here.

SUBDURAL HAEMATOMA

In this investigation subdural haematoma occurred more commonly in African (8\%) than in White patients $(4.6 \%)$. There were six patients with an acute subdural haematoma, five of whom were Black and one a Coloured patient. Evidence of recent head injury was found in five cases, and consisted of lacerations and bruising of the scalp which in only one case was particularly obvious. These five cases were relatively young, none had any evidence of predisposing cortical atrophy, and all died between one and four days after admission. It is highly probable that four patients were admitted with existing subdural haematoma since they were noted either to have cerebral lesions of some kind on admission, or had lapsed into coma by the next day. One patient probably developed his haematoma after admission as he had been assessed as fully conscious on admission, but found dead on the fourth day. The likely course of events in this group of patients was that assault was provoked by their confused and aggressive behaviour, which in turn was symptomatic of a toxic psychosis. Sufficient information was obtainable to make such a diagnosis in three of the cases. The remaining patient with an acute subdural haematoma developed the lesion long after admission to hospital for post-traumatic psychosis, and this was the only case where cortical atrophy was present and there was no history or evidence of recent trauma.

There were six patients with chronic and two with subacute subdural haematomas. The patients belonged to an older age group than those with acute subdural haematomas. Duration of hospitalisation before death was much longer than in the acute group with six patients developing their haematoma a considerable time after admission. Cortical atrophy was present in six cases, but in only one case was there evidence of recent trauma.

The incidence of undiagnosed cases of subdural haematoma was high (13 out of 14). The only case which was diagnosed antemortem was admitted in semi-coma with multiple abrasions of the scalp and head.

The development of subdural haematoma in patients who were already in hospital was sometimes found to have been associated with the onset of additional mental symptoms or neurological signs or both, but this did not necessarily lead to the correct diagnosis. In the elderly a cerebrovascular accident was not infrequently held responsible.

In other cases the development of a subdural haematoma went unnoticed, the first indication being a terminal seizure, or sudden unexplained death. Development of haematoma in the psychotic or senile patient is easily missed. Neither a fall, other trauma nor the classical fluctuation in level of consciousness need be conspicuous, and in most cases was not. Cases admitted with a chronic subdural haematoma may also present diagnostic difficulties, for example, a Black female of 65 years was admitted with a mild hemiparesis, confusion, disorientation, and restless and noisy behaviour. No history was available and the diagnosis of arteriosclerotic dementia was not unreasonable. In some of the cases the presence of neurological signs was perhaps too quickly attributed to cardiovascular disease. If the antemortem diagnosis of subdural haematoma is to be overlooked less frequently an increased awareness of its likelihood and a high degree of vigilance are necessary.

\section{CEREBRAL NEOPLASM}

This was the second most common type of intracranial space-occupying mass, but cases numbered less than half those of subdural haematoma. The number of undiagnosed cases was five out of six, and they serve to illustrate the diagnostic difficulties which exist in psychotic patients with cerebral neoplasm. There were two patients with an oligodendroglioma, and these were typical examples of the sort of case which is misdiagnosed 
because of prominent mental symptoms and lack of neurological signs until a very late stage, or misinterpretation of any neurological signs present. Both cases had undergone neurological investigation, and in neither case did examination reveal the presence of a cerebral tumour. Typical schizophrenic symptoms were present in one patient, and in the other symptoms were attributed to arteriosclerotic brain disease. A Black epileptic patient was found on admission to be demented. A history was obtained that about one year previously he had a brain tumour removed, but a recurrence was not apparently considered. At necropsy a large meningioma was found in the previous operation site. Another Black patient was in hospital for only six days before his death, and cerebral metastases from a bronchogenic carcinoma no doubt contributed to his confusional state. A Black patient with hydrocephalus, epilepsy, and defective mental development was found to have an angiolipoma (hamartoma) in the region of the fourth ventricle which was no doubt responsible for obstruction of the cerebrospinal fluid pathways. In some cases a higher index of suspicion at an earlier stage might have led to detection of cerebral neoplasm, but such examples as these present obvious problems in diagnosis. Perusal of the mental symptoms displayed in these cases supports the general opinion that the psychiatric symptoms accompanying intracranial masses are not of particular localising value or specific help, and indeed may even be misleading.

\section{PARASITIC TAPEWORM CYSTS}

There were two cases where this lesion was found at necropsy. Both cases were epileptic, and in neither was the presence of tapeworm cysts diagnosed during life.

The clinical diagnosis of cerebral tapeworm cysts may be difficult. Most cases are not in a mental hospital and signs and symptoms commonly include those of raised intracranial pressure, hydrocephalus, localising signs of an intracranial mass or epilepsy. Such cases are investigated and diagnosed in a neurological unit. Epilepsy is not considered the most common effect of cerebral tapeworm cyst (Proctor, 1964), nor are tapeworm cysts in the brain the most common cause of epilepsy.

\section{TUBERCULOMA}

There were three cases with tuberculoma at necropsy. None survived more than 10 days after admission to hospital. The antemortem diagnosis of organic brain disease and possibly tuberculous meningitis was considered in two patients, while one had no obvious neurological signs and a diagnosis of toxic psychosis had been made. Psychiatric symptoms were generally unhelpful in making a diagnosis of organic brain disease. Mental symptoms reported were non-specific, and disorientation and confusion were present in all three cases. Restless and violent behaviour and objective hallucinosis was noted in two. Disease was far advanced by the time these patients were referred to a mental hospital, and yet from examination of the histories it appeared that medical opinion was only sought by the relatives because of mental symptoms, and in two of the cases "refusal to eat." Although these patients were wrongly referred to mental hospital, there would at this late stage have been little difference in outcome. It is not known whether any of these patients had previous treatment for tuberculosis, but it is important to note that mental symptoms were prominent at a late stage of the illness, and led to referral to a mental hospital. Neurological signs were only detected at a terminal stage of the illness and certainly had not dominated the clinical picture.

INTRACRANIAL ABSCESS

There were two cases with brain abscess at necropsy. The antemortem diagnosis was correctly made in one patient who developed an extradural abscess after drainage of a haematoma. The second patient was a Black male referred from a general hospital where he had been treated for pneumonia and mental confusion. The pneumonia responded to treatment, but his mental confusion persisted, and he was, therefore, certified and diagnosed as a possible alcoholic psychosis. On admission he was found to have a mild hemiparesis, and investigation was in progress when he died four days later. At necropsy a massive chronic brain abscess, which had clearly been present for some months, was found in the left frontal lobe. The development of pneumonia had contributed to the diagnostic confusion.

In this investigation the incidence of intracranial space-occupying masses and of undiagnosed cases in a mental hospital was high. It was found more common for cases to be admitted with a spaceoccupying mass than to develop it after hospitalisation. The difficulties in diagnosis of an organic brain lesion in the presence of psychosis are emphasised, and continual awareness of its likelihood is just as important for doctors referring cases to mental hospitals as it is for the psychiatrist admitting them. 
This article is based on some of the findings in an MD Thesis conferred by the University of the Witwatersrand, Transvaal, South Africa. I would like to thank the staff of the Sterkfontein Hospital, Transvaal, for their assistance in obtaining necropsy consents and supplying case records.

\section{References}

Allen, A. M., Moore, M., and Daly, B. (1940). Subdural hemorrhage in patients with mental disease. New England Journal of Medicine, 223, 324-329.

Andersson, P. G. (1970). Intracranial tumours in a psychiatric hospital material. Acta Psychiatrica Scandinavica, 46, 213-224.

Froman, C., and Lipschitz, R. (1970). Demography of tumours of the CNS among the Bantu (African) population of the Transvaal. Journal of Neurosurgery, 32, 660-664.

Proctor, N. S. F. (1955). Intracranial tumours and cysts in the South African Bantu. In Proceedings of the Second International Congress of Neuropathology. Excerpta Medica: Amsterdam.

Proctor, N. S. F. (1964). Tapeworm cyst infestation of the central nervous system. Medical Proceedings, 10, 168-171.

Reef, H., Lipschitz, R., and Block, J. (1958). Neurological disorders at Baragwanath Hospital: survey. Medical Proceedings, 4, 292-299.

Selecki, B. (1964). Cerebral midline tumours involving the corpus callosum among mental hospital patients. Medical Journal of Australia, 2, 954-959.

Selecki, B. (1965). Intracranial space-occupying lesions among patients admitted to mental hospitals. Medical Journal of Australia, 1, 383-390. 